\title{
Análisis de impacto presupuestal de la vacunación contra COVID-19 en América Latina
}

\author{
Alejandra Taborda R, ${ }^{1}$ Diego Alejandro Murillo, ${ }^{1}$ Carolina Moreno $L,{ }^{1}$ Paula Andrea Taborda $R,{ }^{1}$ \\ Marcela Fuquen, ${ }^{1}$ Paula Andrea Díaz, ${ }^{2}$ y Darío Londoño ${ }^{3}$
}

Forma de citar

Taborda A, Murillo DA, Moreno C, Taborda PA, Fuquen M, Díaz PA et al. Análisis de Impacto presupuestal de la vacunación contra COVID-19 en América Latina. Rev Panam Salud Publica. 2022;46:e5. https://doi.org/10.26633/ RPSP.2022.5

RESUMEN Objetivo. Estimar el impacto presupuestal de la vacunación contra COVID-19 en seis países de América
Latina: Argentina, Brasil, Chile, Colombia, México y Perú, durante el periodo 2021-2022.
Métodos. Se evaluaron las vacunas de Sinopharm (BBIBP-CorV), Janssen (JNJ-78436735), Instituto de Gama-
leya (Gam-COVID-Vac), Sinovac (CoronaVac), CanSino (Convidecia), AstraZeneca (Vaxzevria), Moderna
(mRNA-1273) y Pfizer (BNT162b2), según disponibilidad para cada país. Se adoptó la perspectiva del sistema
de salud, de manera que solo se incluyeron costos médicos directos. El horizonte temporal se adoptó teniendo
en cuenta los tiempos de implementación de cada plan de vacunación, excluyendo menores de 16 años y
gestantes. Se incluyeron los siguientes costos: costo de la vacunación y aplicación, costos de la hospitalización
general aislamiento, cuidado intermedio e intensivo. Se compararon dos escenarios de vacunación: 1) Población
que desea vacunarse (según las encuestas nacionales) y 2) Población que debería vacunarse (total susceptible
de vacunación). Los costos agregados para cada escenario de vacunación se compararon con el escenario de
no vacunación. Adicionalmente, se realizaron análisis de sensibilidad determinísticos y probabilísticos.
Resultados. Los diferentes esquemas de vacunación contra COVID-19 disponibles en América Latina gene-
ran ahorros potenciales que oscilan entre USD 100 y USD 1500 millones de dólares por país para el período
$2021-2022$, asumiendo que se logra implementar en su totalidad el plan de vacunación previsto en cada país.
Conclusiones. La vacunación contra COVID-19 es una estrategia que además de reducir la morbilidad y
mortalidad para Latinoamérica, genera ahorros potenciales para los sistemas de salud en la región.

Palabras clave Vacunación; coronavirus; SARS-CoV-2; costos de la atención en salud; América Latina.

La enfermedad COVID-19 causada por el virus SARS-CoV-2, fue declarada pandemia por la Organización Mundial de la Salud (OMS) en marzo de 2020, al tiempo que gran parte de los gobiernos mundiales empezaron a implementar medidas de distanciamiento físico, confinamiento y otras medidas de bioseguridad en un intento por disminuir la velocidad en el contagio y evitar un colapso de los sistemas sanitarios (1). A noviembre 21 de 2021 se reportan más de 143 mil casos por millón de habitantes en el mundo, con alrededor de 2316 defunciones por millón de habitantes a causa de esta enfermedad (2).

Diversos países que presentaron una primera ola de contagios a causa del virus, están actualmente experimentado terceras olas o un nuevo aumento en el número de casos, sin que hayan logrado una disminución total desde el comienzo local de la pandemia (3). Lo anterior, a pesar de que se avanza en el proceso de aplicación de vacunas contra COVID-19 en el mundo, el

\footnotetext{
Sapyens SAS BIC. Bogotá, Colombia $\bowtie$ Alejandra Taborda R, alejataborda@ gmail.com

2 Facultad Nacional de Salud Pública. Universidad de Antioquia, Medellín,

Colombia.
} 
cual inició el 8 de diciembre de 2020 (4) en el Reino Unido y que a 21 de noviembre ha alcanzado la cifra de alrededor de 7780 millones de dosis aplicadas en el mundo, y el $66 \%$ de la población latinoamericana cuenta con al menos una dosis recibida (5).

Los esfuerzos públicos y privados por desarrollar vacunas seguras y efectivas tienen pocos precedentes. De hecho, a mayo de 2021 se reportan 287 vacunas candidatas contra la COVID-19, de las cuales 102 están en fase clínica y 185 en fase pre-clínica. De las vacunas que se encuentran en fase clínica, 63 se encuentran entre las fases 1 y 2, 34 entre las fases 2 y 3, en donde destacan la de Sinopharm (BBIBP-CorV), Janssen (JNJ78436735) y la del Instituto de Gamaleya (Gam-COVID-Vac) que se encuentran en la fase 3. La de Sinovac (CoronaVac), CanSino (Convidecia), AstraZeneca (Vaxzevria), Moderna (mRNA-1273) y Pfizer (BNT162b2), se encuentran en la fase 4 (6).

En este contexto, los países enfrentan el reto de disponer de los recursos necesarios para garantizar la adquisición y aplicación de las vacunas en su población. Para ello, el análisis de impacto presupuestal (AIP) puede tener gran relevancia para los tomadores de decisión, ya que la información que arroja permite dimensionar el esfuerzo financiero o los ahorros potenciales dentro de procesos de inclusión de tecnologías en salud. Siendo una herramienta de apoyo para las etapas del proceso presupuestal, toda vez que este permite identificar implicaciones económicas para un gobierno al momento de decidir financiar una tecnología en salud (7), en este caso la implementación de su programa de vacunación contra el COVID-19.

Se ha evidenciado que la vacunación contra COVID-19 en contextos geográficos diferentes a Latinoamérica es una estrategia costo-efectiva. En EEUU por ejemplo, Kholi, Maschio, Becker y Weinsten (8) desarrollaron un Modelo de Markov, para estimar los costos médicos directos y las muertes relacionadas con COVID-19 en una población de pacientes mayores de 65 años, costo por AVAC ganado < USD 50 000. Estos resultados son similares a los obtenidos por Wang et al (9) en Taiwán, para tres vacunas contra COVID-19: BNT162b2 (Pfizer-BioNTech), mRNA-1273 (Moderna), y AZD1222 (Oxford-AstraZeneca), donde su principal resultado es que la vacunación masiva contra COVID-19 disminuye la cantidad de vidas pérdidas por la pandemia, y reduce los costos directos e indirectos en comparación al escenario sin vacunación. En Reino Unido también se evaluó la costó efectividad de los programas de vacunación contra
COVID-19 y las medidas de cuarentena y distanciamiento físico, poniendo a la vacunación como el mejor escenario (10).

Considerando la necesidad de la vacunación contra COVID-19 para disminuir el impacto negativo de la enfermedad en la salud de la población y el uso de recursos sanitarios, se realizó un análisis de impacto presupuestal (AIP) de las vacunas contra COVID-19 en Latinoamérica, desde la perspectiva del sistema de salud o gobierno para Argentina, Brasil, Chile, Colombia, México y Perú.

\section{MATERIALES Y MÉTODOS}

El AIP permite estimar las consecuencias financieras de la adopción de una nueva intervención sanitaria. En otras palabras, el AIP explica cómo un cambio en la combinación de medicamentos y terapias usadas para el tratamiento de una enfermedad particular afectará la trayectoria del gasto en esa condición (11). Para realizar un AIP se estructuran dos escenarios: el actual y el nuevo. El actual considera el tratamiento que está disponible en este momento para el manejo de la condición de salud definida, mientras el nuevo considera la implementación de la nueva tecnología, ambos calculados sobre la misma población objetivo.

La perspectiva de análisis fue del gobierno (sistema de salud), para Argentina, Brasil, Chile, Colombia, México y Perú. El análisis desde esta perspectiva tiene implicaciones directas sobre el financiamiento público y la planeación de los sistemas sanitarios. Se adoptó como escenario actual la no vacunación y el tratamiento que se da a pacientes con COVID-19 que requieren internación hospitalaria, y como escenario nuevo la aplicación de la vacuna para la población susceptible a vacunación, la cual se refiere a toda la población que requieren vacunación a excepción de las gestantes y los menores de 16 años, siguiendo las recomendaciones que se dan desde la evidencia para la población a la fecha de corte. La figura 1 resume la estructura del AIP propuesto.

El costo bajo el escenario actual para el país i, está dado por los costos directos médicos de la población susceptible a vacunar de acuerdo a la siguiente fórmula:

$$
\text { Costo Escenario Actual } \mathrm{i}_{i}=P_{i} E_{i} C_{i}
$$

Para el cálculo del impacto presupuestal de la vacunación en cada país se utilizaron las siguientes variables:

\begin{tabular}{|c|c|c|}
\hline Variable & Notación & Variación del índice \\
\hline Población susceptible a vacunar en el país $i$ & $P_{i}$ & i=Argentina, Brasil, Chile, Colombia, México, Perú \\
\hline $\begin{array}{l}\text { Proporción de la población susceptible que se quiere vacunar } \\
\text { en el país } i\end{array}$ & $R_{i}$ & i=Argentina, Brasil, Chile, Colombia, México, Perú \\
\hline $\begin{array}{l}\text { Proporción de población susceptible que requiere } \\
\text { hospitalización por COVID-19 en el país } i\end{array}$ & $E_{i}$ & i=Argentina, Brasil, Chile, Colombia, México, Perú \\
\hline $\begin{array}{l}\text { Costo promedio de hospitalización de enfermos moderados y } \\
\text { severos por COVID-19 en el país } i\end{array}$ & $C_{i}$ & i=Argentina, Brasil, Chile, Colombia, México, Perú \\
\hline Market share de la vacuna $j$ en el país $i$ & $M_{j}^{i}$ & $\begin{array}{l}\text { i=Argentina, Brasil, Chile, Colombia, México, Perú } \\
j=1,2, \ldots, n \text {, donde } n \text { es el número de medicamentos distribuidos como vacunas contra } \\
\text { COVID-19 }\end{array}$ \\
\hline Costo de adquisición de la dosis de la vacuna $j$ en el país $i$ & $C_{j}^{i}$ & $\begin{array}{l}\text { i=Argentina, Brasil, Chile, Colombia, México, Perú } \\
j=1,2, \ldots, n \text {. }\end{array}$ \\
\hline Costo de aplicación por dosis de vacuna en el país $i$ & $A_{j}$ & i=Argentina, Brasil, Chile, Colombia, México, Perú \\
\hline $\begin{array}{l}\text { Número de dosis requeridas de la vacuna j para completar el } \\
\text { esquema de vacunación }\end{array}$ & $D_{j}$ & $j=1, \ldots, n$ \\
\hline Efectividad de la vacuna $j$ & $E f_{j}$ & $J=1, \ldots, n$ \\
\hline
\end{tabular}


FIGURA 1. Análisis de Impacto Presupuestal de la vacunación contra COVID-19 por cada país.

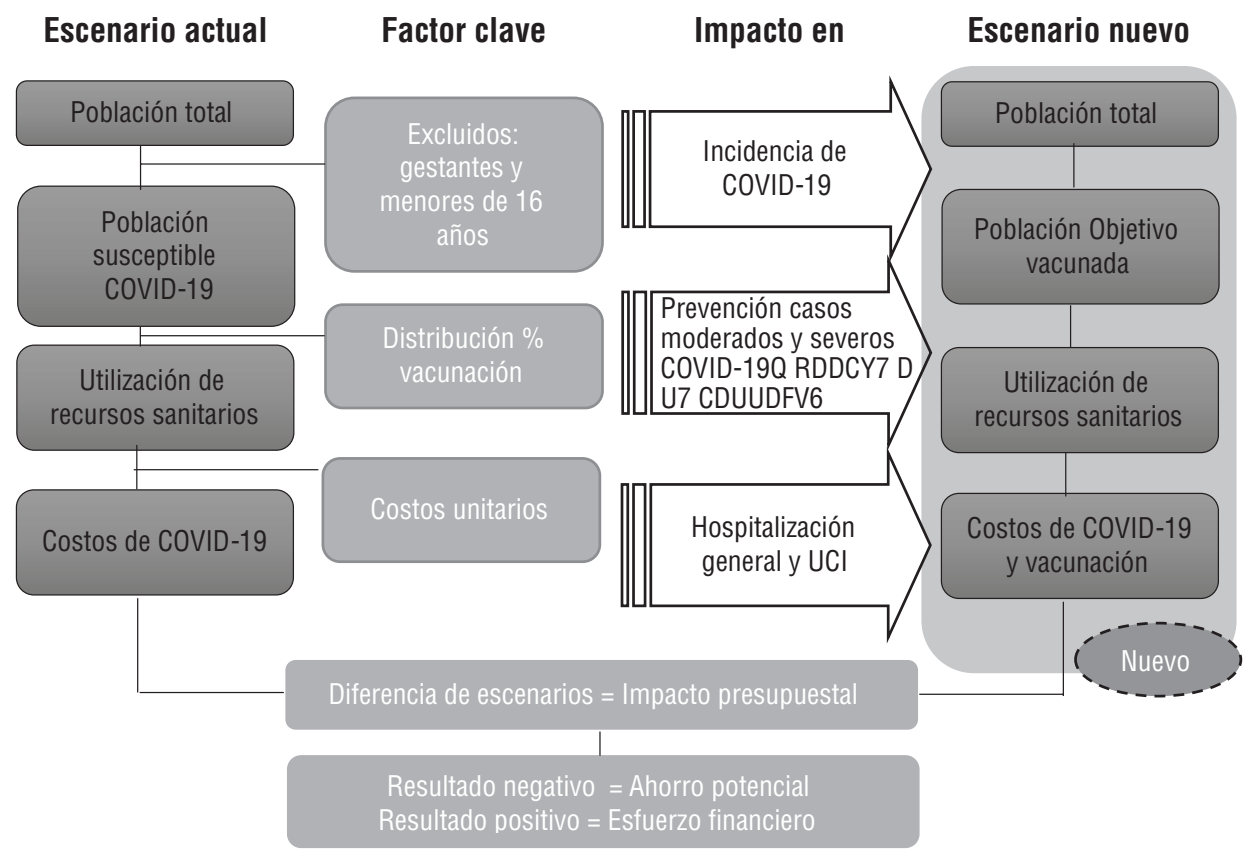

Fuente: elaboración propia con base en Mauskopf Josephine A. et al (11).

El costo bajo el escenario nuevo de la vacunación para el país $i$, está dado por los costos de vacunación de la población que se quiere vacunar, los costos médicos de la población en que la vacuna no es efectiva, y los costos médicos de la población que no se quiere vacunar, de acuerdo a la siguiente fórmula:

\section{Costo Escenario $\mathrm{Nuevo}_{i}$}

$$
:=P_{i} R_{i}\left[\sum_{j=1}^{n} M_{j}^{i}\left[\left(P_{j}^{i}+A_{i}\right) D_{j}+\left(1-E f_{j}\right) E_{i} C_{i}\right]\right]+P_{i}\left(1-R_{i}\right) E_{i} C_{i}
$$

Finalmente, el AIP para el país $i$ está dado por la siguiente expresión:

$$
A I P_{i}:=\text { Costo Escenario Nuevo }_{i} \text { - Costo Escenario } \text { Actual }_{i}
$$

Si el resultado es positivo, se interpreta como el esfuerzo financiero que se debe realizar para la financiación del nuevo tratamiento, y si el resultado es negativo, indicará que se obtendrá un ahorro de ser implementado el nuevo tratamiento.

\section{Parámetros del modelo}

Se presentan todos los parámetros y supuestos metodológicos, así como las fuentes empleadas para cada etapa del proceso del AIP:

Población: en el escenario actual, la población total se obtuvo de las oficinas de estadística de cada país, a partir de la cual se calcularon los casos totales de infectados por COVID-19 que requirieron hospitalización. Para el escenario de vacunación, se tuvo en cuenta la población objetivo susceptible de vacunación por COVID-19 y la población que desea vacunarse contra el virus, según encuestas reportadas para cada país. La primera se tomó de los planes de vacunación de los países de análisis, con corte a 31 de mayo, con exclusión de menores de 16 años y gestantes. La información de población que desea vacunarse es clave para el análisis del impacto presupuestal, pues si bien no afecta el monto total que debe invertirse en la adquisición de las vacunas (que se estima sobre la población susceptible), sí incide sobre los costos agregados de la estrategia, toda vez que la población no vacunada permanecería susceptible de infectarse y por tanto requerir hospitalización (al menos mientras se logra la inmunidad de rebaño).

Costos: para el escenario actual, el costo y días promedio de hospitalización por COVID-19 para casos moderados y severos se extrajo de la siguiente forma: para Argentina y Chile, el costo de hospitalización fue suministrado por hospitales de alta complejidad, los cuales solicitaron mantener en confidencialidad los datos y la fuente de los datos. Para Perú, los costos de hospitalización para este país se tomaron de prensa nacional (12) y los días promedio de estancia se tomaron de un estudio realizado para un hospital público de Lima (13). Para Colombia, los datos se tomaron del Ministerio de Salud y Protección Social (14). Para México y Brasil los costos se tomaron de reportes de prensa reconocidos (15).

Para el escenario nuevo, se obtuvo el número de las vacunas negociadas de los planes de vacunación de cada uno los países. En Brasil y Perú, se encontró una cifra definida de dosis negociadas mediante el mecanismo COVAX sin especificar de qué farmacéutica serían las dosis. Por ello, se hizo una aproximación de las vacunas que podrían entregarse con base en el histórico de acuerdos que ha hecho COVAX (100 millones de dosis con AstraZeneca y 40 millones de dosis con Pfizer) (16). 
El costo de cada una de las vacunas se obtuvo principalmente de información reportada en informes de prensa, toda vez que, debido a los acuerdos de confidencialidad suscritos entre los países y las farmacéuticas, esta información no se encuentra disponible. Para el costo de aplicación de las vacunas, se tomó como referencia un valor promedio estándar de 7 USD con sus respectivas variaciones de costo intramural y extramural estimado por el Ministerio de Salud de Colombia (17).
Eficacia de las vacunas: para el análisis de eficacia y seguridad, se tomaron las nueve vacunas más relevantes en cuanto a participación del número de dosis negociadas por cada uno de los países. Así pues, se incluyeron para el análisis las vacunas de Pfizer, AstraZeneca, Moderna, Janssen Cilag, CanSino Biologics, Sinopharm, Sputnik V y Sinovac. Los datos de eficacia y seguridad de las vacunas analizadas se tomaron de los resultados interinos de los estudios clínicos pivótales de cada una (18-24) (cuadro 1).

CUADRO 1. Parámetros del Análisis de Impacto Presupuestal para los países estudiados 2021-2022 (AIP)+

\begin{tabular}{|c|c|c|c|c|c|c|}
\hline & Argentina & Brasil & Chile & Colombia & México & Perú \\
\hline \multicolumn{7}{|c|}{ Población } \\
\hline Población candidata a vacunar (a) & 24000000 & 149510023 & 15000000 & 34234649 & 96100000 & 26379565 \\
\hline Porcentaje de personas que se quieren vacunar (b) & $76 \%$ & $89 \%$ & $74 \%$ & $72 \%$ & $80 \%$ & $63 \%$ \\
\hline $\begin{array}{l}\text { Proporción de personas que requirieron hospitalización por COVID-19 en } \\
\text { un año (c) }\end{array}$ & $0,3 \%$ & $0,1 \%$ & $0,7 \%$ & $1,0 \%$ & $0,5 \%$ & $1,8 \%$ \\
\hline \multicolumn{7}{|c|}{ Número de dosis de vacunas negociadas (a) } \\
\hline Total dosis Pfizer & - & 210139370 & 10000000 & 10000000 & 34150250 & 24272579 \\
\hline Total dosis AstraZeneca & 22000000 & 254762400 & 14400000 & 10000000 & 85912800 & 23428571 \\
\hline Total dosis Moderna & - & - & - & 10000000 & - & - \\
\hline Total dois Janssen Cilag & - & 38000000 & 4000000 & 9000000 & - & - \\
\hline Total dosis Sputnik V & 25000000 & 10000000 & - & - & 24000000 & - \\
\hline Total dosis Sinovac & - & 130000000 & 60000000 & 7500000 & - & - \\
\hline Total dosis Sinopharm & 1000000 & - & - & - & - & 1000000 \\
\hline Total dosis CanSino Biologics & - & - & 1800000 & - & 35000000 & - \\
\hline \multicolumn{7}{|c|}{ Costos (Dólares) (d) } \\
\hline Costo promedio por dosis Pfizer & - & 19 & 12 & 12 & 19,5 & 40 \\
\hline Costo promedio por dosis AstraZeneca & - & 3,2 & 4 & 8 & 2,8 & 4 \\
\hline Costo promedio por dosis Moderna & - & - & - & 29 & - & - \\
\hline Costo promedio por dosis Janssen Cilag & - & 10 & 10 & 9 & - & - \\
\hline Costo promedio por dosis Sputnik V & 9,5 & 10 & - & - & 10 & - \\
\hline Costo promedio por dosis Sinovac & - & 10,3 & 14 & 17 & - & - \\
\hline Costo promedio por dosis Sinopharm & 30 & - & - & - & - & 33 \\
\hline Costo promedio por dosis CanSino Biologics & - & - & 4 & - & 4 & - \\
\hline Costo de aplicación & 8 & 8 & 7 & 6 & 3 & 7 \\
\hline Costo por día de hospitalización general COVID-19 (e) & 322,0 & 424,1 & 1020,0 & 379,9 & 451,4 & 754,0 \\
\hline Costo por día de hospitalización cuidado intermedio y UCI COVID-19 (e) & 764,0 & 780,0 & 1923,0 & 925,5 & 2500,0 & 1819,1 \\
\hline \multicolumn{7}{|c|}{ Eficacia de las vacunas } \\
\hline & $\begin{array}{l}\text { Casos } \\
\text { moderados }\end{array}$ & Casos severos & & & $\begin{array}{l}\text { Casos } \\
\text { moderados }\end{array}$ & Casos severos \\
\hline Pfizer (18) & NR & $95 \%$ & Janssen Cilag (19) & & $67 \%$ & $95 \%$ \\
\hline AstraZeneca (20) & $76 \%$ & $100 \%$ & Sputnik V (21) & & $100 \%$ & $92 \%$ \\
\hline Moderna (22) & NR & $94 \%$ & Sinovac (22) & & $100 \%$ & $51 \%$ \\
\hline CanSino Biologics (23) & $90 \%$ & $65 \%$ & Sinopharm (24) & & $100 \%$ & $78 \%$ \\
\hline
\end{tabular}

(+) Si desea ampliar la información utilizada para el análisis, por favor comunicarse con el autor de correspondencia.

(a) Tomados de los planes vigentes de vacunación de cada país al 31 de mayo de 2021 (25-30)

(b) Según encuestas nacionales de cada país entre diciembre de 2020 y mayo de 2021.

(c) Estimación a partir de fuentes oficiales de casos reportados en cada país.

(d) Reportes en medios de comunicación reconocidos para cada país.

(e) Los costos de hospitalización por COVID-19 fueron suministrados por hospitales de referencia para cada país.

Nota: valores están expresados en millones de dórales americanos (USD), el cambio de moneda local a dólares se realizó el 30 de junio mediante "Google Finance".

Fuente: elaboración propia

Para cada país, se calculó en Excel el AIP considerando la población total susceptible y la población que desea vacunarse. También se realizó un análisis de sensibilidad probabilístico en Python con 1000 iteraciones (31). Se utilizaron distribuciones beta para simular la proporción de población que se quiere vacunar, la probabilidad de hospitalización en caso de contraer COVID-19, y la proporción que cada vacuna actualmente adquirida representa en el total de vacunas adquiridas, teniendo en cuenta que la proporción simulada de cada vacuna debe normalizarse luego para que la suma de proporciones sea igual a 1 . Por otra parte, se utilizó una distribución gamma para simular los costos de hospitalización, dado que estos costos son incurridos por una proporción muy baja de la población susceptible, pero suelen ser muy altos en caso de ser incurridos. También se 
asumió que el costo de aplicación por dosis se distribuye uniformemente entre 2 y 8 dólares, y que el resto de los parámetros son deterministas e iguales a los valores utilizados en el estudio.

Para determinar los parámetros de las distribuciones beta, se asumieron las proporciones dadas por los valores utilizados en el estudio. Por ejemplo, para simular la proporción de población que se quiere vacunar en Argentina, se utilizó los parámetros $\propto=760$ y $\beta=240$.

Para simular la distribución gamma de los costos de hospitalización, se utilizan los parámetros

$$
\begin{aligned}
k & =\frac{\text { Costos de hospitalización esperados }^{2}}{\text { Varianza de costos de hospitalización }} y \theta \\
& =\frac{\text { Varianza de costos de hospitalización }}{\text { Costos de hospitalización esperados }} .
\end{aligned}
$$

Dado que no hay información sobre la varianza de los costos de hospitalización, esta varianza se estimó con la proporción de casos moderados para cada país y los costos de hospitalización esperados para casos moderados y para casos severos, de acuerdo con la siguiente expresión $\left(\operatorname{Var}(X)=E\left(X^{\wedge} 2\right)-E(X)^{\wedge} 2\right)$ :

Varianza de costos de hospitalización $=$ Prop $_{\text {mod }} \times \mathrm{CHosp}^{2}{ }_{\text {mod }}$ + Prop $_{\text {sev }} \times \mathrm{CHosp}_{\text {sev }}^{2}-$ Costos de hospitalización esperados ${ }^{2}$

donde para cada país, Prop $_{\text {mod }}$ y Prop $_{\text {sev }}=1-$ Prop $_{\text {mod }}$ son las proporciones de casos moderados y severos respectivamente, $\mathrm{y}$
CHosp mod $^{\prime}$ CHosp sev $^{\prime}$ son los costos promedio de hospitalización de casos moderados y severos de COVID-19 respectivamente

\section{RESULTADOS}

En el cuadro 2 se presentan los costos agregados para cada escenario según lo invertido en vacunación y uso de recursos de acuerdo a la efectividad de las mismas, así como el gasto per cápita estimado para los seis países analizados. Los países deben invertir en la vacunación según la población susceptible o que debería vacunarse. Los países con más gasto en vacunación son los de mayor población total susceptible a vacunar, como es el caso de Brasil con USD 4582 millones, seguido de México con USD 1512 millones. En cuanto al gasto per cápita estimado para la vacunación contra COVID-19, se evidencia un promedio de USD 36 por persona para los países analizados, sin embargo, se destacan con el valor más bajo México (USD 20) y el más alto Perú (USD 59).

El resultado de impacto presupuestal del caso base, se presenta en la figura 2, donde se observa que la estrategia de vacunación genera ahorros en todos los países, que pueden oscilar entre 100 y 1500 millones de dólares.

Por otra parte, los resultados del análisis de sensibilidad (figura 3) indican que los costos de hospitalización tienen un efecto sobre el ahorro incremental, lo que se explica por la reducción del número de hospitalizaciones en caso de vacunación. Es importante recalcar que la variación vertical del AIP para un mismo nivel de costos de hospitalización promedio representa el rango de valores en que se espera que varíe el AIP, siempre que el resto de los parámetros se hayan simulado

\begin{tabular}{|c|c|c|c|c|c|c|}
\hline \multirow[b]{2}{*}{ Argentina } & \multirow{2}{*}{$\begin{array}{l}\text { Costos estimados } \\
\text { Vacunación }\end{array}$} & \multicolumn{2}{|c|}{$\begin{array}{l}\text { Escenario actual } \\
\text { (Sin vacunar) }\end{array}$} & $\begin{array}{c}\text { Escenario nuevo } 1 \text { (Población } \\
\text { que desea vacunarse) }\end{array}$ & $\begin{array}{c}\text { Escenario nuevo } 2 \text { (Población } \\
\text { que debería vacunarse) }\end{array}$ & \multirow{2}{*}{$\begin{array}{c}\text { Gasto per cápita estimado para } \\
\text { vacunación * } \\
\$ 31\end{array}$} \\
\hline & & $\$$ & - & $\$ 562020000$ & $\$ 739500000$ & \\
\hline & No efectividad vacuna & $\$$ & - & $\$ \quad 86274939$ & $\$ 113519656$ & \\
\hline & No vacunados & $\$$ & - & $\$ 563926881$ & $\$ \quad-$ & \\
\hline & Total casos COVID-19 & \multicolumn{2}{|c|}{$\$ 2349695336$} & $\$ 1212221819$ & $\$ 853019656$ & \\
\hline \multirow[t]{4}{*}{ Brasil } & Vacunación & $\$$ & - & $\$ 4582462943$ & $\$ 5148834767$ & $\$ 34$ \\
\hline & No efectividad vacuna & $\$$ & - & $\$ 737396974$ & $\$ 828535926$ & \\
\hline & No vacunados & $\$$ & - & $\$ 785399340$ & $\$ \quad-$ & \\
\hline & Total casos COVID-19 & \multicolumn{2}{|c|}{$\$ 7139993997$} & $\$ 6105259256$ & $\$ 5977370693$ & \\
\hline \multirow[t]{4}{*}{ Chile } & Vacunación & $\$$ & - & $\$ 428396009$ & $\$ \quad 575587583$ & $\$ 38$ \\
\hline & No efectividad vacuna & $\$$ & - & $\$ 308980394$ & $\$ 417541073$ & \\
\hline & No vacunados & $\$$ & - & \$ 317143493 & $\$$ & \\
\hline & Total casos CoVID-19 & \multicolumn{2}{|c|}{$\$ 1219782667$} & $\$ 1054519896$ & $\$ 993128656$ & \\
\hline \multirow[t]{4}{*}{ Colombia } & Vacunación & $\$$ & - & $\$ 878132735$ & $\$ 1216250326$ & $\$ 36$ \\
\hline & No efectividad vacuna & $\$$ & - & $\$ 273950395$ & $\$ 379432681$ & \\
\hline & No vacunados & $\$$ & - & \$ 626827637 & $\$$ & \\
\hline & Total casos COVID-19 & \multicolumn{2}{|c|}{$\$ 2254775674$} & $\$ 1778910768$ & $\$ 1595683006$ & \\
\hline \multirow[t]{4}{*}{ México } & Vacunación & $\$$ & - & $\$ 1512426007$ & $\$ 1890532509$ & $\$ 20$ \\
\hline & No efectividad vacuna & $\$$ & - & $\$ 222662946$ & $\$ 278328682$ & \\
\hline & No vacunados & $\$$ & - & $\$ \quad 627864867$ & $\$$ & \\
\hline & Total casos COVID-19 & \multicolumn{2}{|c|}{ \$ 3139324335} & $\$ 2362953820$ & $\$ 2168861191$ & \\
\hline \multirow[t]{4}{*}{ Perú } & Vacunación & $\$$ & - & $\$ 980979567$ & $\$ 1557110424$ & $\$ 59$ \\
\hline & No efectividad vacuna & $\$$ & - & $\$ 1115100164$ & $\$ 1770000261$ & \\
\hline & No vacunados & $\$$ & - & $\$ 1656404267$ & $\$ \quad-$ & \\
\hline & Total casos COVID-19 & \multicolumn{2}{|c|}{$\$ 4476768290$} & \$ 3752483998 & \$ 3327110684 & \\
\hline
\end{tabular}

CUADRO 2 . Resultados agregados y per cápita por escenarios en todos los países estudiados, 2021-2022

*Estimado a partir del escenario nuevo 2 y la población total a vacunar para cada país.

Nota: valores están expresados en millones de dórales americanos (USD) 
de forma cercana a la realidad. Para cada país, un aumento en los costos de hospitalización promedio por paciente para COVID-19 hace que el ahorro utilizando la vacunación sea potencialmente mayor, lo que se explica por la eficacia y seguridad de las vacunas, independientemente de las combinaciones de los otros parámetros.

\section{DISCUSIÓN}

La salud pública en Latinoamérica y el mundo enfrenta un reto histórico a raíz de la pandemia COVID-19, poniendo en riesgo la población que históricamente ha mostrado inequidades en los sistemas de salud y las políticas sociales (31).

FIGURA 2. Ahorros o esfuerzo financiero comparativo, caso base en los países estudiados (en millones de dólares)

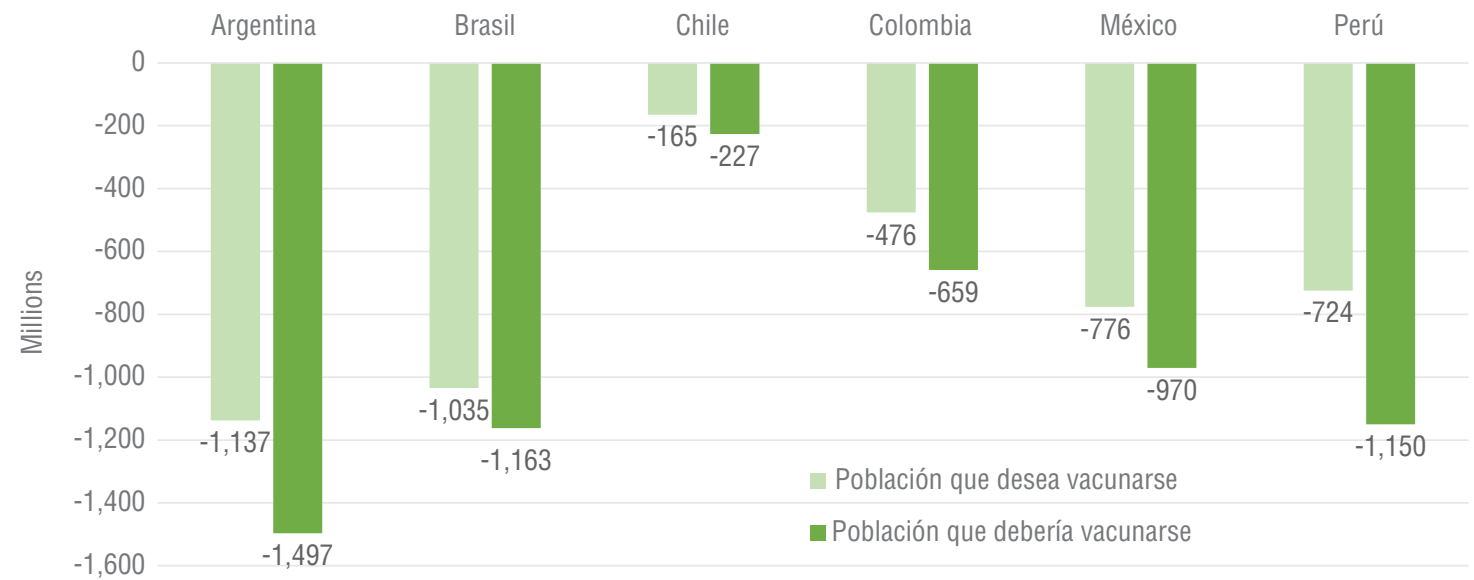

Nota: valores están expresados en millones de dórales americanos (USD)

FIGURA 3. Análisis de sensibilidad probabilístico del análisis de impacto presupuestal de la vacunación contra COVID-19 en países de América Latina, 2021-2022

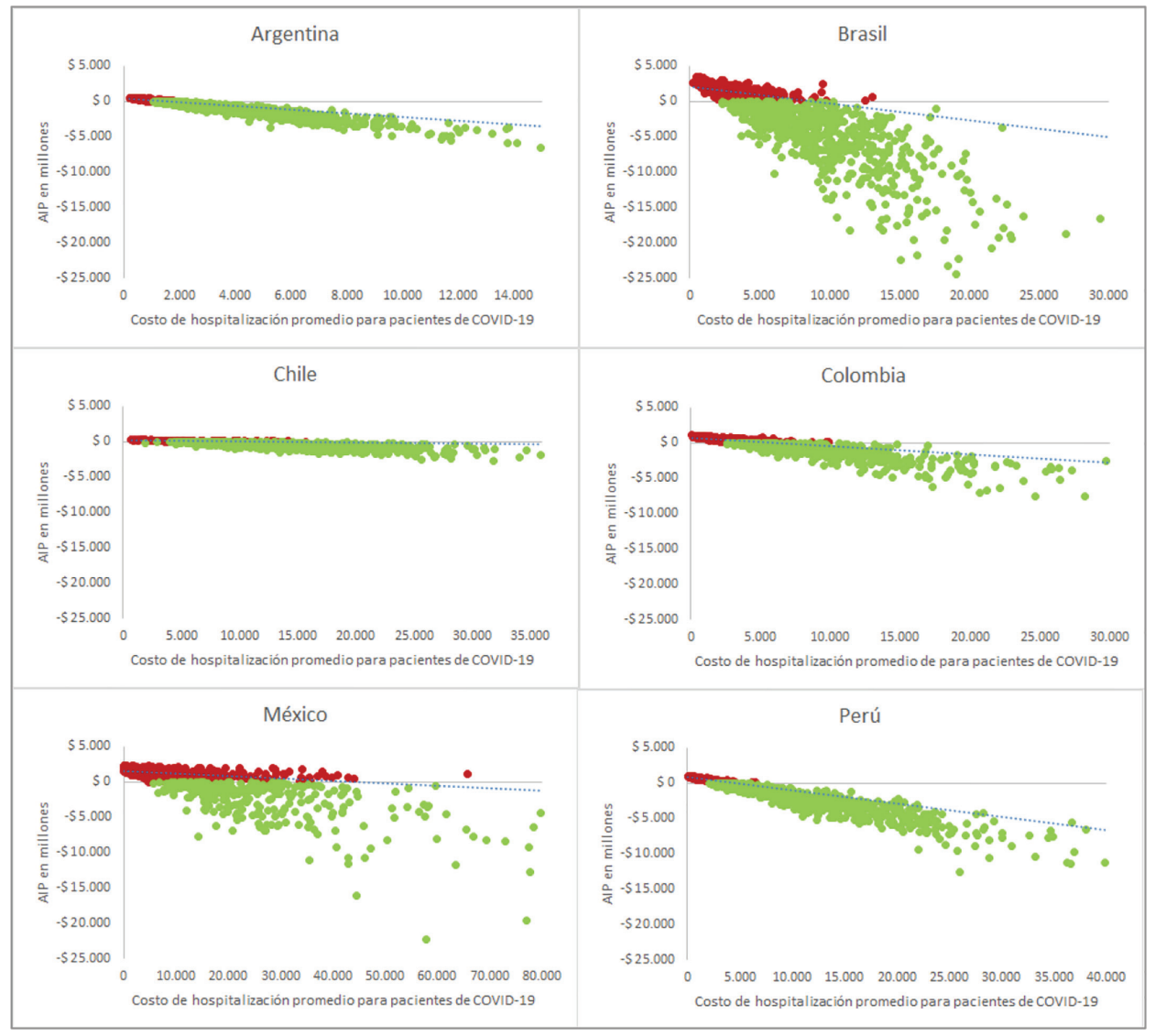

Nota: valores están expresados en millones de dórales americanos (USD) 
Históricamente la vacunación ha sido una de las intervenciones más efectivas para controlar y erradicar enfermedades, y en el contexto actual, se ha convertido en la mejor herramienta para combatir la pandemia (32). Con este estudio, se evidencian las acciones particulares de cada contexto en los planes de vacunación para hacer frente y mitigar los efectos en la salud poblacional, como la población priorizada en fases de aplicación, los tiempos de respuesta según el inicio de casos y la capacidad de negociar las vacunas y garantizar cobertura universal.

Todos los países de Latinoamérica incluidos en el análisis de impacto presupuestal mostraron un ahorro potencial significativo por incluir la estrategia de vacunación en la población general. La vacunación contra COVID-19 no sólo evita casos graves y muertes por esa causa, sino que también genera ahorros potenciales para los sistemas de salud. La diferencia de los ahorros estimados para cada contexto, se pueden explicar principalmente en función de tres determinantes: 1) la cantidad de la población a vacunar, cada país ha mostrado a través de encuestas nacionales que en promedio el 30\% de la población no se desea vacunar; 2) la distribución de cuotas de mercado de las vacunas, según el costo negociado por cada país y su eficacia evaluada; y 3) los mayores costos son los asociados a la hospitalización, a las tarifas y el promedio de días de estancia en pacientes moderados, severos y críticos.

La pandemia de COVID-19 ha revelado la importancia de la planeación y generación de evidencia en salud para la gestión de recursos hospitalarios en el corto y mediano plazo para responder a las necesidades de la población. Se han evidenciado limitaciones frente a la disponibilidad de información en costos, como días de hospitalización general, cuidados intermedios y UCI por evento, estas limitaciones son críticas para la planeación presupuestal en salud frente a la pandemia. Adicional a esto, existe mucha variación dentro de cada país en torno a la información. El aumento de costos depende de la condición clínica y severidad de los pacientes, el costo promedio día de hospitalización general fue de USD 559 y de USD 1452 para cuidado intermedio y crítico en la región.

Los resultados muestran un beneficio real en términos de reducción de los costos esperados de hospitalización, sin embargo, existen otros beneficios y ahorros potenciales de costos no considerados por el estudio, los cuales pudieran incrementar aún más los beneficios de las políticas de vacunación contra COVID-19. En primer lugar, ha sido necesario obtener una mayor inversión para la salud, aumento de la capacidad instalada y contratación de personal cualificado para lograr el funcionamiento, así como mejoras en los procesos de atención los sistemas de salud, esto bajo la garantía de sostenibilidad (33); en segundo lugar, la pérdida de productividad y el gasto de bolsillo de las familias por causa de COVID-19 puede ser mucho menor con los efectos de la vacunación, siempre y cuando se apliquen los esquemas completos, se acompañe de medidas de bioseguridad y distanciamiento social.

La disponibilidad de vacunas para Latinoamérica ha sido menor que en países desarrollados, lo cual hace que los retos para la negociación sean mucho mayores. Las vacunas Pfizer, Moderna, y AstraZeneca se desarrollaron durante el primer año de la pandemia, un tiempo récord considerando que usualmente puede tardar una década, que abarca una fase preclínica, 4 fases clínicas, y una fase de revisión (28). El corto tiempo en el desarrollo de las vacunas contra COVID-19 y demanda en el nivel global de la vacunación, que afectan claramente su costo de producción.

Las diferencias en Latinoamérica en términos de capacidades económicas, sociales y políticas llevaron a condiciones particulares de negociación entre las farmacéuticas y los gobiernos, enmarcadas dentro de acuerdos de confidencialidad, generando ocultamiento y asimetrías de información, entre otras fallas de mercado, y haciendo más difícil el acceso universal y oportuno a las diferentes tecnologías para controlar y manejar el COVID-19 $(34,35)$.

El volumen de vacunas que necesitan adquirir los países demarca la capacidad de negociar el precio con las compañías farmacéuticas. La confidencialidad en los acuerdos de precios entre las compañías farmacéuticas y los países, favorecen brechas para países con limitada capacidad económica y de adquisición de volumen de las vacunas. Las diferencias entre los países en términos de capacidad económica y necesidad en volumen de adquisición de vacunas impactarán el valor final para cada país. Para el caso de la vacuna de Pfizer se evidenció una variación de precio entre USD 12 y USD 40 entre los países, para la Sinovac entre USD 10 y USD 17, la menos costosa fue AstraZeneca con una variación entre USD 2,8 y USD 8 en los países analizados de la región.

Se espera que la propagación del virus a nivel mundial continúe en el mediano y largo plazo. Sin embargo, se desconoce la periodicidad y endemicidad que se va a enfrentar y los costos que implica tanto para COVID-19 como para las patologías relevantes en cada país. Este panorama que nos revela enormes retos para la salud pública, y especialmente para Latinoamérica son mucho mayores, en la medida que los gobiernos y sistemas de salud no muestran fortaleza desde la perspectiva del capital social, debilitando las instituciones y sus acciones articuladas. Nuestro gran reto como región es crear sinergias y unir esfuerzos regionales para lograr coberturas de vacunación universales a costos razonables.

Dentro de las limitaciones del estudio, se debe mencionar que no se cuenta con registros oficiales de precios de las vacunas por acuerdos de confidencialidad entre las farmacéuticas y los distintos países. Además, los planes de vacunación de los países se van ajustando en la medida que se cuente con nueva evidencia científica o la inclusión de nuevas vacunas disponibles con registros sanitarios en el mercado. Es posible que en el mediano plazo se puedan incluir gestantes y menores de 16 años, lo cual cambiar las estimaciones de impacto presupuestal y posibles ahorros en los países latinoamericanos analizados en este estudio, esta población equivale entre el $6 \%$ y $10 \%$ para los países seleccionados de la región.

En conclusión, la vacunación contra COVID-19 genera ahorros potenciales que oscilan entre 100 y 1500 millones de dólares por país para el año 2021, siempre y cuando se logre implementar en su totalidad el plan de vacunación previsto en cada país; pese a la incertidumbre que existe sobre los costos de COVID-19, el análisis de sensibilidad probabilístico mostró una tendencia significativa y dominante al ahorro en todos los países. En este contexto, cobra especial relevancia la predisposición a vacunarse que se observa en cada uno de ellos, ya que una mayor implementación del plan de vacunación generará mayores ahorros a los países.

Contribución de los autores. Todos los autores concibieron el estudio original, diseñaron los modelos, recolectaron los 
parámetros, analizaron los datos, interpretaron los resultados, escribieron y revisaron el manuscrito. Todos los autores revisaron y aprobaron la versión final.

Agradecimiento. Agradecemos a Andrea Perinetti de Chile, Rita Mattielo de Brasil, Eduardo Goicochea de México y a Victor
Ynami de Perú, por ayudarnos a complementar y validar la información sobre costos de hospitalización de los países.

Declaración. Las opiniones expresadas en este manuscrito son responsabilidad del autor y no reflejan necesariamente los criterios ni la política de la $R P S P / P A J P H$ y/o de la OPS

\section{REFERENCIAS}

1. OPS/OMS. La OMS caracteriza a COVID-19 como una pandemia. [Internet]. Washington DC:Organización Panamericana de la Salud,organización Mundial de la Salud; 2020;1-13 [citado 2021 Nov 24]. Disponible en: https://www.paho.org/es/ noticias /11-3-2020-oms-caracteriza-covid-19-como-pandemia

2. Our World in Data. Coronavirus Pandemic (COVID-19) - the data - Statistics and Research [Internet]. Oxford:University of Oxford; 2021 [citado 2021 Jun 29]. Disponible en : https:/ / ourworldindata. org/coronavirus-data

3. OPS/OMS. Actualización Epidemiológica Enfermedad por coronavirus (COVID-19) [Internet] Washington DC: Organización Panamericana de la Salud; 2021 [citado 2021 Jun 27]. Disponible en: https://www.paho.org/es/file/89978/ download?token=PfVQYU4L

4. BBC News. Reino Unido comienza vacunación masiva contra la covid-19: cómo es el proceso y qué se puede esperar [Internet]. 2020 [citado 2021 Jun 27]. Disponible en: https://www.bbc.com/ mundo/noticias-55212779

5. Our World in Data. Coronavirus (COVID-19) Vaccinations - Statistics and Research [Internet] Oxford:University of Oxford;2021. 2021 [citado 2021 Nov 24]. Disponible en: https://ourworldindata.org/ covid-vaccinations?country=OWID_WRL

6. OMS. COVID-19 vaccine tracker and landscape [Internet]Ginebra:Organización Mundial de Salud;2021[citado 2021 Jun 24]. Disponible en: https://www.who.int/publications/m/item/ draft-landscape-of-covid-19-candidate-vaccines

7. IETS. Manual para la elaboración de análisis de impacto presupuestal [Internet]. 2014 [Citado 2021 Jun 24]. Disponible en: https:// www.minsalud.gov.co/sites/rid/Lists/BibliotecaDigital/RIDE/ INEC/IETS/manual-metodologico-analisis-impacto-presupuestal. pdf

8. Kohli M, Maschio M, Becker D, Weinstein MC. The potential public health and economic value of a hypothetical COVID-19 vaccine in the United States: Use of cost-effectiveness modeling to inform vaccination prioritization. Vaccine. 2021;39(7):1157-64.doi. org /10.1016/j.vaccine.2020.12.078

9. Wang WC, Fann JC, Chang RE, Jeng YC, Hsu CY, Chen HH, Liu $\mathrm{JT}$, Yen AM. Economic evaluation for mass vaccination against COVID-19. J Formos Med Assoc. 2021 Jun;120 Suppl 1:S95-S105. doi: 10.1016/j.jfma.2021.05.020.

10. Sandmann FG, Davies NG, Vassall A, Edmunds WJ, Jit M; Centre for the Mathematical Modelling of Infectious Diseases COVID-19 working group. The potential health and economic value of SARS-CoV-2 vaccination alongside physical distancing in the UK: a transmission model-based future scenario analysis and economic evaluation. Lancet Infect Dis. 2021 Jul;21(7):962-974. doi: 10.1016/S1473-3099(21)00079-7. Erratum in: Lancet Infect Dis. 2021 Oct;21(10):e302.

11. Mauskopf JA, Sullivan SD, Annemans L, Caro J, Mullins CD, Nuijten M, et al. Principles of good practice for budget impact analysis: Report of the ISPOR Task Force on Good Research Practices - Budget Impact Analysis. Value Heal. 2007;10(5):336-47. doi: 10.1111/j.1524-4733.2007.00187.x.

12. Períodico el Búho. Coronavirus y realidad: los imposibles costos de internarse en una clínica privada [Internet]. 2020 [citado 2021 Sep 27]. Disponible en: https://elbuho.pe/2020/06/ peru-coronavirus-y-la-pobreza-los-imposibles-costos-en-unaclinica-privada/

13. Casquino KV, Venero AE, Galvez JCQ. Mortalidad y factores pronósticos en pacientes hospitalizados por COVID-19 en la Unidad de Cuidados Intermedios de un hospital público de Lima, Perú. Horiz Médico. 21(1), e1370. Disponible en: https://www.horizon temedico.usmp.edu.pe/index.php/horizontemed/article / view /1370/911

14. Ministerio de de Salud y Protección Social. Se define valores de referencia a pagar por servicios UCI de covid-19 [Internet]Bogóta: Ministerio de de Salud y Protección Social; 2020 [citado 2021 Sep 27]. Disponible en: https://www.minsalud.gov.co/Paginas/Sedefine-valores-de-referencia-a-pagar-por-servicios-UCI-de-covid-19 .aspx

15. Períodico el Contribuyente México. Éstos son los costos de atención médica en el IMSS en 2021 [Internet]. [citado 2021 Sep 27]. Disponible en: https://www.elcontribuyente.mx/2021/01/estosson-los-costos-de-atencion-medica-en-el-imss-en-2021/

16. Organización Mundial de la Salud. COVAX anuncia un nuevo acuerdo y previsiones sobre las primeras entregas de vacunas [Internet]Ginebra:OMS;2021 [citado 2021 Jun 24]. Disponible en: https://www.who.int/es/news/item/22-01-2021-covax-announces-new-agreement-plans-for-first-deliveries

17. Ministerio de Saud y Protección Social. Resolución No. 166 de 2021 [Internet]. [Citado 2021 Jun 24]. Bogóta: Ministerio de Saud y Protección Social; 2021. Disponible en: https://www.minsalud.gov. co/Normatividad_Nuevo/Resoluci\%C3\%B3n\%20No.\%20166\%20 de\%202021.pdf

18. Kyriakidis NC, López-Cortés A, González EV, Grimaldos AB, Prado EO. SARS-CoV-2 vaccines strategies: a comprehensive review of phase 3 candidates. NPJ Vaccines. 2021 Feb 22;6(1):28. doi: 10.1038/ s41541-021-00292-w.

19. Sadoff J, Gray G, Vandebosch A, Cárdenas V, Shukarev G, Grinsztejn B, et al. ENSEMBLE Study Group. Safety and Efficacy of Single-Dose Ad26.COV2.S Vaccine against Covid-19. N Engl J Med. 2021 10;384(23):2187-2201. doi: 10.1056/NEJMoa2101544.

20. Ramasamy MN, Minassian AM, Ewer KJ, Flaxman AL, Folegatti PM, Owens DR, et al. Oxford COVID Vaccine Trial Group. Safety and immunogenicity of ChAdOx1 $\mathrm{nCoV}-19$ vaccine administered in a prime-boost regimen in young and old adults (COV002): a single-blind, randomised, controlled, phase 2/3 trial. Lancet. 2021 Dec 19;396(10267):1979-1993. doi: 10.1016/S0140-6736(20)32466-1.

21. Logunov DY, Dolzhikova I V., Shcheblyakov D V., Tukhvatulin AI, Zubkova O V., Dzharullaeva AS, et al. Safety and efficacy of an rAd26 and rAd5 vector-based heterologous prime-boost COVID-19 vaccine: an interim analysis of a randomised controlled phase 3 trial in Russia. Lancet . 2021;397(10275):671-81

22. WHO Strategic Advisory Group of Experts (SAGE) on Immunisation. Evidence Assessment: Sinovac/CoronaVac COVID-19 vaccine. 2021;32. Disponible en: https://cdn.who.int/media/docs/ default-source/immunization/sage/2021/april/5_sage29apr2021_ critical-evidence_sinovac.pdf?sfvrsn=2488098d_5

23. CanSino Biologics. NMPA Accepts the Application for Conditional Marketing Authorization of CanSinoBIO's COVID-19 Vaccine ConvideciaTM [Internet]. 2021 [citado 2021 Jun 29]. Disponible en: http:/ / www.cansinotech.com/html/1/179/180/651.html

24. SAGE Working Group on COVID-19 vaccines. Evidence assessment: Sinopharm/BBIBP COVID-19 vaccine. 2021; Disponible en: https://cdn.who.int/media/docs/default-source/immunization/ sage/2021/april/2_sage29apr2021_critical-evidence_sinopharm. pdf?sfvrsn=3dfe32c1_5

25. Ministerio de Salud de Argentina. Plan Estratégico Para La Vacunación Contra La Covid-19 En La república de Argentina. 2020; Disponible en: https://www.argentina.gob.ar/sites/default/files/ 
coronavirus-vacuna-plan-estrategico-vacunacion-covid-19-diciembre -2020.pdf

26. Ministério da Saúde, Secretaria de Vigilância em Saúde (BR). Operacionalização Da Vacinação Contra a Covid-19. 4ta edición Brazilia; 2021. Disponible en: https://www.gov.br/saude/pt-br/ media/pdf/2021/janeiro/29/PlanoVacinaoCovid_ed4_15fev21_ cgpni_18h05.pdf

27. Ministerio de Salud. Subsecretaría de salud pública de Chile. Planificación: vacunación contra sars-cov-2. 2021; Disponible en: https://www.minsal.cl/wp-content/uploads/2021/02/ Planificaci\%C3\%B3n-vacunaci\%C3\%B3n-contra-SARSCoV-2-02-02-2021.pdf

28. Ministerio de de Salud y Protección Social. Departamento Nacional de Planeación Ministerio de Hacienda y Crédito Público Instituto de Evaluación Tecnológica en Salud. Plan Nacional de Vacunación contra el SARS-CoV-2. 2021;Bogóta: Ministerio de de Salud y Protección Social; 2021. Disponible en: https:/ / www.minsalud.gov.co/ sites/rid/Lists/BibliotecaDigital/RIDE/VS/pnv-contra-covid-19. pdf

29. Gobierno de México. Política Nacional de Vacunación contra el virus SARS-CoV-2. 1ra edición. Ciudad de México: Gobierno de México; 2020. Disponible en: https://www.pediatria.gob.mx/ archivos/covid-1.pdf

30. Ministerio de Salud de Perú. Plan Nacional Actualizado contra la COVID-19.pdf. 2021. Disponible en: https://cdn.www.gob.pe/ uploads/document/file/1805113/Plan\%20Nacional\%20Actualizado $\% 20$ contra $\% 201$ \% $\% 20$ COVID-19.pdf
31. Mar J, Antoñanzas F, Pradas R, Arrospide A. Los modelos de Markov probabilísticos en la evaluación económica de tecnologías sanitarias: Una guía práctica. Gac Sanit. 2010;24(3):209-14.

32. Barrientos-Gutiérrez T, Alpuche-Aranda C, Lazcano-Ponce E, Pérez-Ferrer C, Rivera-Dommarco J. La salud pública en la primera ola: una agenda para la cooperación ante Covid-19. Salud Publica Mex . 2020;62(5):598-606.doi.org/10.21149/11606

33. OMS. Vacunas e inmunización: ¿qué es la vacunación? [Internet] Ginebra: Organización Mundial dela Salud; 2020 [citado 2021 Jun 29]. Disponible en: https://www.who.int/es/news-room/q-a-detail/ vaccines-and-immunization-what-is-vaccination

34. Evenet SJ, Fritz J. El COVID-19 como renovado detonador del acaparamiento y la depredación comercial: ¿el multilaterismo en coma? Glob Trade Alert Rep [Internet]. 2021 [citado 2021 Jun 27]; Disponible en: www.globaltradealert.org/reports

35. ForoALC2030. Cuarta reunión virtual del Foro de los Países de América Latina y el Caribe sobre el Desarrollo Sostenible 2021 [Internet] 2021 [citado 2021 Jun 27]. Disponible en: https:/ / foroalc2030.cepal. org/2021/es

Manuscrito recibido el 01 de julio del 2021. Aceptado para su publicación, tras revisión, el 13 de octubre del 2021.

\section{Analysis of budgetary impact of COVID-19 vaccination in Latin America}

ABSTRACT Objective. To estimate the budgetary impact of COVID-19 vaccination in six Latin American countries: Argentina, Brazil, Chile, Colombia, Mexico, and Peru, during the 2021-2022 biennium.

Methods. Vaccines from Sinopharm (BBIBP-CorV), Janssen (JNJ-78436735), Gamaleya Institute (GamCOVID-Vac), Sinovac (CoronaVac), CanSino (Convidecia), AstraZeneca (Vaxzevria), Moderna (mRNA-1273), and Pfizer (BNT162b2) were evaluated, according to their availability in each country. The health system perspective was adopted, so that only direct health care costs were included. The time horizon adopted took into account the implementation times of each vaccination plan, excluding children under 16 years of age and pregnant persons. The following costs were included: cost of vaccination/vaccine administration and costs of hospitalization (general isolation, stepdown care, and intensive care). Two vaccination scenarios were compared: 1) population wanting to be vaccinated (according to national surveys); and 2) population that should be vaccinated (total population susceptible to vaccination). The aggregate costs for each vaccination scenario were compared with the no-vaccination scenario. Deterministic and probabilistic sensitivity analyses were also performed.

Results. The different COVID-19 vaccination regimens available in Latin America generate potential savings ranging from USD 100 million to USD 1.5 billion per country for the 2021-2022 biennium, assuming that the vaccination plan proposed for each country is fully implemented.

Conclusions. COVID-19 vaccination is a strategy that not only reduces morbidity and mortality in Latin America, but also generates potential savings for health systems in the region.

Keywords Vaccination; coronavirus; SARS-CoV-2; health care costs; Latin America. 


\section{Análise do impacto orçamentário da vacinação contra a COVID-19 na América Latina}

RESUMO Objetivo. Estimar o impacto orçamentário da vacinação contra a COVID-19 em seis países da América Latina: Argentina, Brasil, Chile, Colômbia, México e Peru, no período 2021-2022.

Métodos. Foram avaliadas as vacinas da Sinopharm (BBIBP-CorV), Janssen (JNJ-78436735), Instituto Gamaleya (Gam-COVID-Vac), Sinovac (CoronaVac), CanSino (Convidecia), AstraZeneca (Vaxzevria), Moderna (mRNA-1273) e Pfizer (BNT162b2), conforme a disponibilidade para cada país. Adotou-se a perspectiva do sistema de saúde, de forma que só foram incluídos custos médicos diretos. O horizonte temporal foi adotado levando em consideração os tempos de implementação de cada plano de vacinação, excluindo crianças menores de 16 anos e gestantes. Foram incluídos os seguintes custos: custos de vacinação e aplicação, custos gerais de hospitalização, isolamento, e cuidados intermediários e intensivos. Compararam-se dois cenários de vacinação: 1) população disposta a se vacinar (com base em pesquisas nacionais) e 2) população que deveria ser vacinada (total elegível de vacinação). Os custos agregados para cada cenário de vacinação foram comparados com o cenário de não vacinação. Além disso, foram realizadas análises de sensibilidade determinísticas e probabilísticas.

Resultados. Os diferentes esquemas de vacinação contra a COVID-19 disponíveis na América Latina geram economias potenciais entre 100 milhões e 1,5 bilhão de dólares por país para o período 2021-2022, considerando a implementação completa do plano de vacinação previsto em cada país.

Conclusões. A vacinação contra a COVID-19 é uma estratégia que, além de reduzir a morbidade e a mortalidade na América Latina, gera economias potenciais para os sistemas de saúde da região.

Palavras-chave Vacinação; coronavirus; SARS-CoV-2; custos de cuidados de saúde; América Latina. 